\title{
Effects of the Ophthalmic Additive Mannitol on Antimicrobial Activity and Corneal Toxicity of Various Preservatives
}

\author{
Kazunori Inaba, Misa Minami, Mizuki Yamaguchi, Ryoka Goto, Hiroko Otake, \\ Takeshi Kotake, and Noriaki Nagai* \\ Faculty of Pharmacy, Kindai University; 3-4-1 Kowakae, Higashi-Osaka, Osaka 577-8502, Japan \\ Received July 4, 2020; accepted August 24, 2020
}

\begin{abstract}
Ophthalmic preservatives are indispensable in eye drop formulations, but may be toxic to corneal structures. Corneal damage necessitates the discontinuation of treatment with ophthalmic solutions. Therefore, the development of a new and safe preservative system without corneal toxicity is needed. The present study investigated the effects of mannitol on the antimicrobial activities and corneal toxicities of various preservatives using Escherichia coli and a human corneal epithelial cell line (HCE-T cells). The following preservatives were examined: boric acid (BA), benzalkonium chloride (BAC), methyl parahydroxybenzoate (MP), propyl parahydroxybenzoate (PP), sodium chlorite (SC), and zinc chloride (ZC). The antimicrobial activities and HCE-T-cell toxicities of $50 \mu \mathrm{g} / \mathrm{mL} \mathrm{BA}, \mathrm{MP}, \mathrm{PP}, \mathrm{SC}$, and ZC were reduced by a co-treatment with mannitol $(0-300 \mu \mathrm{g} / \mathrm{mL})$. The suppressed antimicrobial activities of BA, MP, PP, and SC by the co-treatment with mannitol were restored by the application of a mannitol content higher than $500 \mu \mathrm{g} / \mathrm{mL}$. In contrast to these 5 preservatives, the addition of mannitol did not affect the antimicrobial activity of BAC and attenuated its HCE-T cell toxicity. Therefore, the balance between the contents of mannitol and preservatives is important in co-treatments. The present results will serve as a guide for the future development of eye drop formulations without corneal toxicity.
\end{abstract}

Key words mannitol; preservative; antimicrobial activity; corneal damage; benzalkonium chloride

\section{Introduction}

Eye drop formulations are packaged under sterile conditions; however, improper use and ambient air are sources of contamination, resulting in ocular infections. ${ }^{1,2)}$ Preservatives are added to maintain the sterility and markedly extend the shelf-life of the formulation. An enhanced shelf-life is convenient and cost effective for eye drops because it allows for the marketing of large bottles. Although preservatives in eye drop formulations reduce the risk of bacterial contamination, they may also be toxic to ocular structures, inducing cellular and molecular damage. In addition, the acute stimulation slowly accumulates, leading to chronic or irreversible damage of clinical relevance, particularly in long-term therapies. These side effects need to be minimized not only for treatment efficacy and compliance, but also to reduce corneal damage and increase safety in clinical practice.

Mannitol is a non-reducing sugar alcohol that is used to decrease pressure in the eyes, such as glaucoma, and lower an elevated intracranial pressure. It is also an osmotic agent. Liu et al. previously reported that mannitol exerted antioxidant effects by increasing the level of catalase, which is decreased by hydrogen peroxide. ${ }^{3)}$ We previously demonstrated that sericin and mannitol attenuated corneal toxicity caused by commercially available timolol maleate eye drops. ${ }^{4,5)}$ The combination of mannitol and preservatives may be a less toxic formulation. Benzalkonium chloride (BAC), parahydroxybenzoate (MP), propyl parahydroxybenzoate (PP), sodium chlorite (SC), boric acid (BA), and zinc chloride (ZC) are preservatives that are frequently used in the ophthalmic field, and are indispensable for safe use and the prevention of secondary infection. In currently marketed ophthalmic solutions, approximately $70 \%$ contain the preservative BAC, approximately
$20 \%$ MP and PP (parabens), and 10\% BA, SC, and ZC. The present study investigated the effects of mannitol on the corneal toxicities and antimicrobial activities of various preservatives using a human corneal epithelial cell line (HCE-T cells) and Escherichia coli.

\section{Experimental}

Materials BA, mannitol (D-mannitol), MP, PP, and ZC were purchased from Wako Pure Chemical Corporation (Osaka, Japan). BAC was obtained from Kanto Chemical Co., Inc. (Tokyo, Japan). SC were purchased from SigmaAldrich Co., LLC. (Tokyo, Japan). Mueller Hinton Broth was obtained from Becton, Dickinson and Company (Fukushima, Japan). Dulbecco's modified Eagle's medium/Ham's F12 (DMEM/F12), Gibco fetal bovine serum (FBS), streptomycin, and penicillin were purchased from Thermo Fisher Scientific Inc. (Tokyo, Japan). Cell count reagent SF was provided by Nacalai Tesque Inc. (Kyoto, Japan). E. coli (ATCC 8739) was provided by the National Institute of Technology and Evaluation (Tokyo, Japan). The immortalized human corneal epithelial cell line (HCE-T cells, RCB No. 1384) used in the present study was donated by RIKEN. All other chemicals used were of the highest purity commercially available.

Preparation of Preservative Solutions Test samples were dissolved in purified water and used to prepare mannitol and various preservatives, which were then filtered through Minisart CE (pore size of $0.20 \mu \mathrm{m}$, Costar, MA, U.S.A.). The concentration of mannitol used in assessments of damage to corneal cells was $1 \mathrm{mg} / \mathrm{mL}$, less than or equal to the maximum tolerated ophthalmological dose. The concentration of preservatives was $50 \mu \mathrm{g} / \mathrm{mL}$, reflecting clinical concentrations.

Antimicrobial Activities of Preservatives The antimi- 
crobial activities of preservatives were measured using $E$. coli according to Japanese Pharmacopoeia (JP) test protocols. ${ }^{6}$ Briefly, six types of preservatives and/or mannitol in sterilized Mueller Hinton Broth were added to sterilized saline containing $10^{8}$ colony-forming units (CFU)/mL of E. coli (1 organism per aliquot), which were then incubated at $35^{\circ} \mathrm{C}$ for $24 \mathrm{~h}$ in a 96-well microplate (AGC TECHNO GLASS Co., Ltd., Shizuoka, Japan). The antimicrobial activity was judged by visual observation according to JP. ${ }^{6}$ The minimal inhibitory concentration (MIC) estimated from the lowest concentration exhibiting antimicrobial activity against E. coli was used in experiments.

Cell Culture and Treatment Corneal damage was assessed in accordance with the method reported by Nagai et ll. $^{4)}$ HCE-T cells were cultured in DMEM/F12 containing $5 \%$ (v/v) heat-inactivated FBS, $100 \mu \mathrm{g} / \mathrm{mL}$ streptomycin, and $100 \mathrm{IU} / \mathrm{mL}$ penicillin. HCE-T cells $\left(1 \times 10^{4}\right.$ cells $)$ were seeded on 96 -well microplates. Saline or $50 \mu \mathrm{g} / \mathrm{mL}(0.005 \%)$ preservative solutions with or without $1 \mathrm{mg} / \mathrm{mL}$ (final concentration) mannitol were added to cell cultures one day after seeding, and cells were stimulated for $0-120 \mathrm{~s}$. After the stimulation, culture medium containing the cell count reagent SF (Nacalai Tesque Inc.) was added, and absorbance (Abs) at $450 \mathrm{~nm}$ was measured using a microplate reader (Bio-Rad Laboratories, Richmond, CA, U.S.A). Cell viability was calculated accord-

Table 1. MIC of Ophthalmic Preservatives against E. coli

\begin{tabular}{cc}
\hline \hline Ophthalmic preservatives & MIC $(\mu \mathrm{g} / \mathrm{mL})$ \\
\hline BA & 4 \\
BAC & 16 \\
MP & 6 \\
PP & 1.25 \\
SC & 5 \\
ZC & 25 \\
Mannitol & 102400
\end{tabular}

The experiments were performed at 3 times.
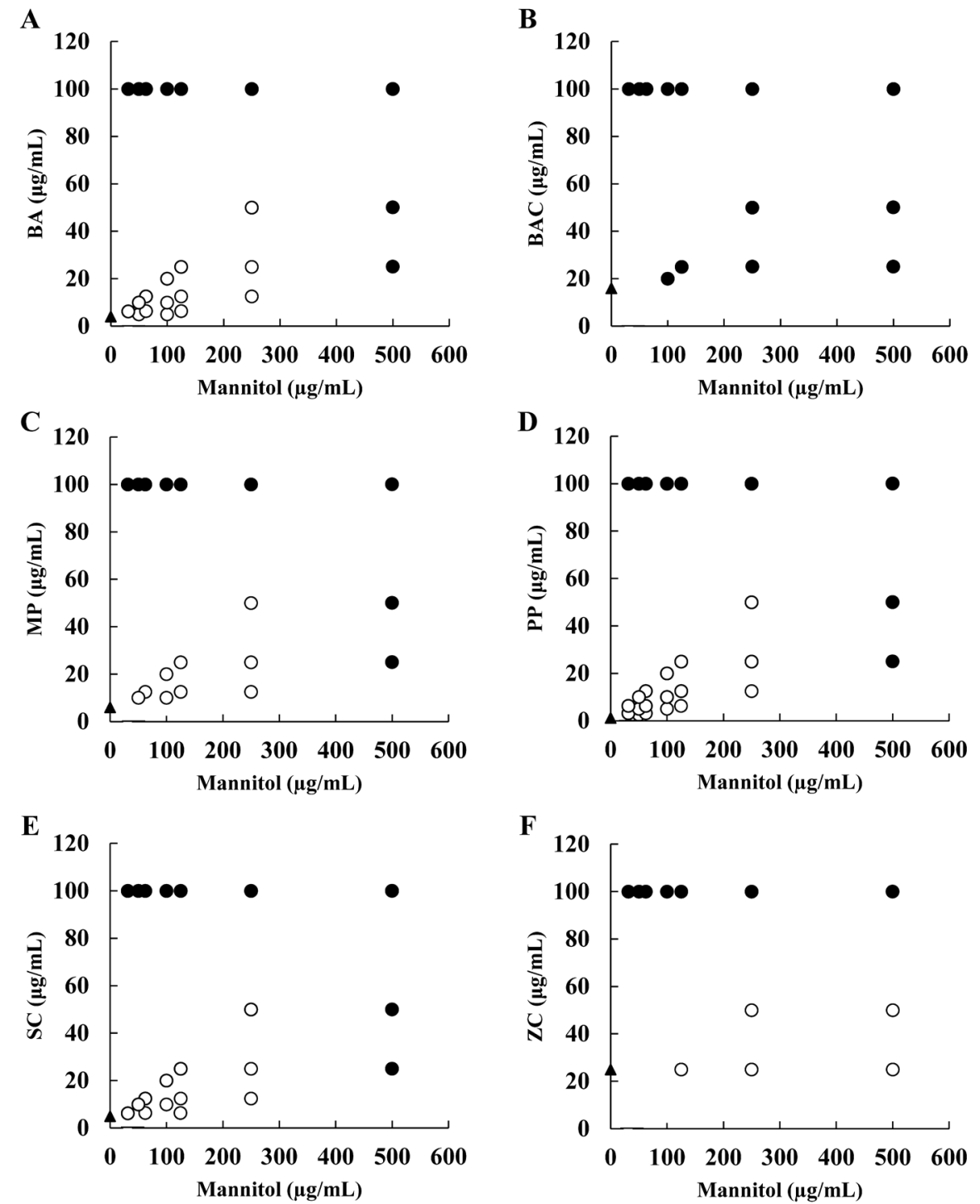

B

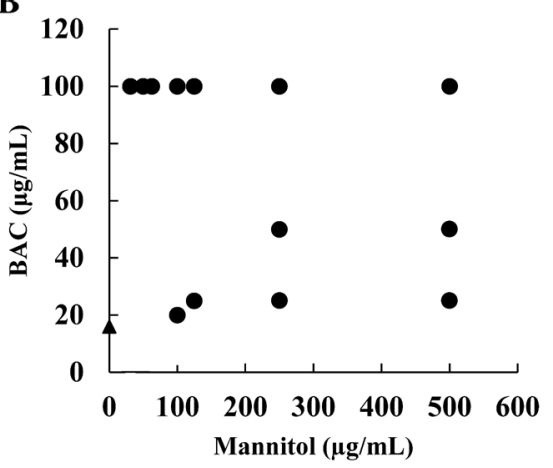

F

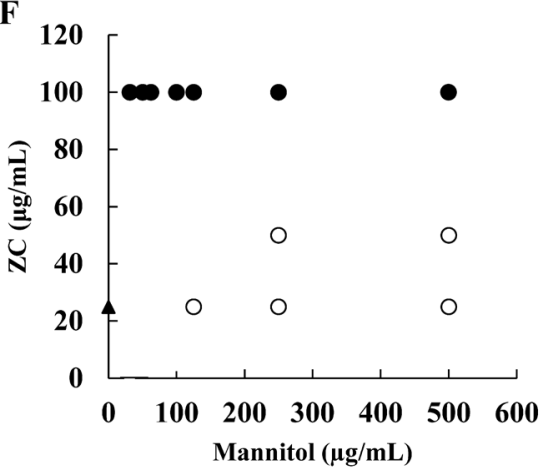

Fig. 1. Effects of Mannitol on Antimicrobial Activities of Various Preservatives in E. coli

E. coli ATCC 8739 were incubated in preservative [BA (A), BAC (B), MP (C), PP (D), SC (E) and ZC (F)] solutions with or without mannitol for 24h. Open circles, groups without antimicrobial activity. Closed circles, groups with antimicrobial activity. Closed triangles, MIC of each preservative. The experiments were performed at 3 times, and the reproducibility has been confirmed. The antimicrobial activities of BA, MP, PP, SC, and ZC were decreased by the co-treatment with mannitol, whereas that of BAC was not. 
ing to equation (Eq.) 1:

$$
\text { Cell viability }(\%)=\mathrm{Abs}_{\text {treatment } /} \mathrm{Abs}_{\text {non-treatment }} \times 100
$$

Statistical Analysis Statistical comparisons were performed using the Student's $t$-test and Dunnett's multiple comparison using JMP (SAS Institute Inc., Cary, NC, U.S.A.). $p<0.05$ was considered to be significant.

\section{Results}

Antimicrobial Activity in E. coli Co-treated with Mannitol and Preservatives Table 1 shows the MIC of the 6 ophthalmic preservatives used in the present study. These preservatives prevented the growth of $E$. coli with MIC in the following order: $\mathrm{ZC}(25 \mu \mathrm{g} / \mathrm{mL})>\mathrm{BAC}(16 \mu \mathrm{g} / \mathrm{mL})>\mathrm{MP}$ $(6 \mu \mathrm{g} / \mathrm{mL})>\mathrm{SC}(5 \mu \mathrm{g} / \mathrm{mL})>\mathrm{BA}(4 \mu \mathrm{g} / \mathrm{mL})>\mathrm{PP}(1.25 \mu \mathrm{g} / \mathrm{mL})$. Figure 1 shows the antimicrobial activities of combinations of mannitol and the 6 preservatives in E. coli. The antimicrobial activities of $\mathrm{BA}, \mathrm{MP}, \mathrm{PP}, \mathrm{SC}$, and $\mathrm{ZC}$ were decreased by the co-treatment with mannitol, and regardless of the mannitol content, the antimicrobial activities of BA, MP, PP, SC, and $\mathrm{ZC}$ were observed when used at concentrations of higher than $100 \mu \mathrm{g} / \mathrm{mL}$. In addition, when the content of mannitol was
$500 \mu \mathrm{g} / \mathrm{mL}$, the suppressed antimicrobial activities of BA, MP, PP, and SC were restored. Furthermore, we measured the antimicrobial activity in the combination of $1 \mathrm{mg} / \mathrm{mL}$ mannitol and preservatives, and the antimicrobial activity were observed in the all preservatives. In contrast to these preservatives, the addition of mannitol did not affect the antimicrobial activity of BAC.

Toxicity in HCE-T Cells Co-treated with Mannitol and Preservatives Figure 2 shows changes in the viability of HCE-T cells treated with the 6 types of preservative solutions (BA, BAC, MP, PP, SC, and ZC) with or without mannitol. The preservatives exhibited toxicity against these cells in the order of $\mathrm{BAC}>\mathrm{SC}>\mathrm{PP}>\mathrm{MP}>\mathrm{ZC}>\mathrm{BA}$. However, the addition of mannitol reduced the toxicities of the preservatives. In the treatment for $120 \mathrm{~s}$, the viabilities of BA-, BAC-, MP-, PP-, SC-, and ZC-treated HCE-T cells co-treated with mannitol were $93.9,58.7,75.7,77.8,80.7$, and $65.7 \%$-fold higher, respectively, than those of HCE-T cells treated with the preservatives only.

\section{Discussion}

Preservatives are indispensable in the preparation of oph-
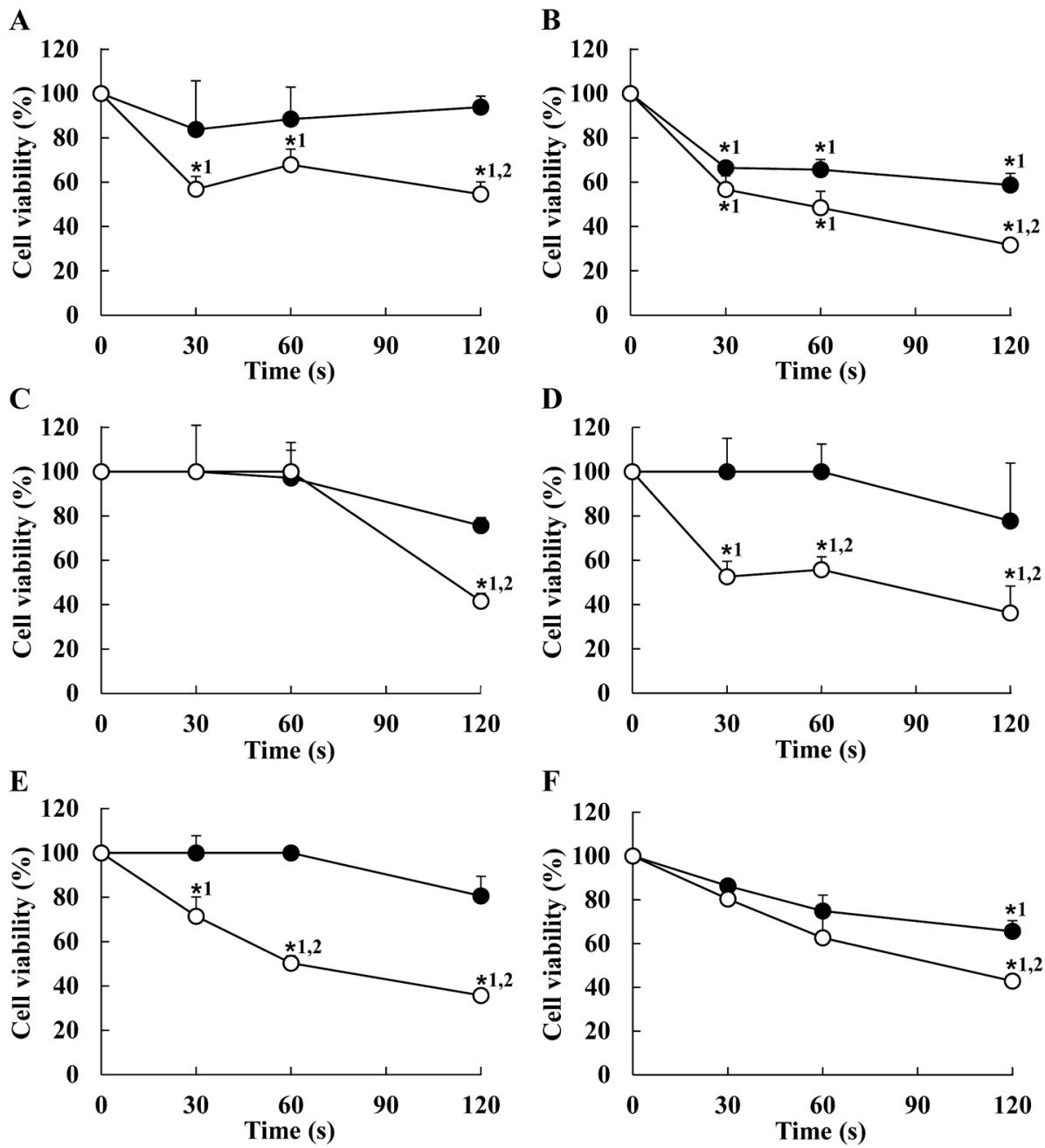

Fig. 2. Preventive Effects of Mannitol on Cell Toxicities of Various Preservatives in HCE-T Cells

HCE-T cells in 96-well microplates were treated with $0.005 \%$ preservative [BA (A), BAC (B), MP (C), PP (D), SC (E), and ZC (F)] solutions with or without $0.1 \%$ mannitol. Open circles, HCE-T cells treated with preservatives. Closed circles, HCE-T cells co-treated with mannitol and preservatives. Data are shown as the means \pm standard error of 3 experiments. ${ }^{* 1} p<0.05, v s$. non-treated HCE-T cells $(0 \mathrm{~s})$ for each category. ${ }^{* 2} p<0.05, v s$. HCE-T cells treated with the preservative for each category. Cell toxicity was induced by the 6 preservatives used in the present study. On the other hand, the co-treatment with mannitol attenuated the cell toxicities of all preservatives. 
thalmic solutions. However, they are toxic to corneal structures, and corneal damage necessitates the discontinuation of therapy for patients requiring the long-term application of ophthalmic solutions. Therefore, the development of a safe and new preservative system without corneal toxicity is needed. We previously reported that mannitol attenuated the corneal toxicity of BAC in HCE-T cells and a rat debrided corneal epithelium. ${ }^{4}$ Furthermore, we previously showed that the transcorneal penetration and anti-intraocular pressure effect in the rabbit instilled with timolol containing $0.5 \%$ mannitol were similar to those of timolol without mannitol. ${ }^{4}$ Therefore, a combination of mannitol and preservatives may be used in ophthalmic formulations without corneal toxicity. In the present study, we investigated the effects of mannitol on the antimicrobial activities of 6 commonly used preservatives. We also examined whether the combination with mannitol decreased the corneal toxicities of these preservatives.

In clinical settings, BA, BAC, MP, PP, SC, and ZC are used as ophthalmic preservatives in eye drop formulations. We selected these 6 preservatives in the present study, and investigated changes in their antimicrobial activities and corneal toxicities when combined with mannitol. Antimicrobial activities were assessed using $E$. coli. The six preservatives all were below the detection limit for $E$. coli after $24 \mathrm{~h}$, and exhibited strong antimicrobial activities against $E$. coli. Therefore, antimicrobial activity may be maintained after $24 \mathrm{~h}$. However, the antimicrobial activity of mannitol was weak, with MIC of $102400 \mu \mathrm{g} / \mathrm{mL}$ (Table 1). We then investigated whether the antimicrobial activities of the 6 preservatives were affected by the combination with mannitol. Previous studies reported the mechanisms underlying the antimicrobial effects of preservatives. BA is an inorganic acid that is used in a wide and diverse array of clinical and industrial applications. Although it is used topically as a bacteriostatic and fungistatic agent, its mechanism of action remains unclear. Furthermore, limited information is currently available on the pharmacology of topically applied BA; however, it is generally considered to be safe for short-term use. ${ }^{7)}$ The mechanisms underlying the antimicrobial effects of parabens (MP and PP) involve the loss of membrane potential by an increase in permeability to membrane ions or the impaired respiratory function of mitochondria. Longer carbon lengths have been shown to reduce cytotoxicity. ${ }^{8,9)}$ Furthermore, SC penetrates bacterial cells and stops gene activity by reacting with nucleotide sulfhydryls, sulfides, and disulfides to disrupt protein synthesis, resulting in strong bactericidal effects. ${ }^{10)} \mathrm{ZC}$ increases the production of hydrogen peroxide, which contributes to its antimicrobial activity. ${ }^{11)}$ In the present study, the combination with mannitol decreased the antimicrobial activities of these preservatives (Fig. 1), but not that of BAC (Fig. 1B). BAC is a quaternary ammonium compound that alters the ionic resistance of cells by intercalating into cellular membranes, resulting in membrane disruption via oxidative stress. In addition, BAC disrupts cell membranes, the organization of intracellular enzyme proteins, and the inhibition of the respiratory system by the adsorption of positively charged atomic groups on the surface of bacterial cells. ${ }^{12-14)}$ In the present study, the reason why the antimicrobial activity of BAC alone was not decreased by the co-treatment of mannitol remains unclear. However, differences in the mechanisms of antimicrobial activity may be involved. On the other hand, regardless of the mannitol content, the antimicrobial activities of BA, MP, PP, SC, and ZC were observed when used at concentrations of higher than $100 \mu \mathrm{g} / \mathrm{mL}$, and the suppressed antimicrobial activities of BA, MP, PP, and SC by the co-treatment of mannitol were restored at a mannitol content higher than that of $500 \mu \mathrm{g} / \mathrm{mL}$. It is important to evaluate the use of preservatives other than BAC, since there are some commercially available eye drops containing mannitol and other preservatives (without BAC) in the clinic, and these results indicate that the balance between the contents of preservatives and mannitol was an important factor contributing to reductions in the antimicrobial activities of BA, MP, PP, and SC. Although, the mannitol has no clear antimicrobial activity at a concentration of $500 \mu \mathrm{g} / \mathrm{mL}$, the mannitol also has weak antimicrobial activity (the MIC is $102400 \mu \mathrm{g} / \mathrm{mL}$, Table 1). Taken together, it is possible that the antimicrobial activity increased due to an additive or synergistic effect. Further studies are needed to evaluate the mechanism of antimicrobial activities in the combination of mannitol and preservatives. In addition, the evaluations of antimicrobial activity using bacteria other than $E$. coli will be important for clarifying the usefulness of preservatives containing mannitol for ophthalmic solutions.

An experimental protocol to clarify corneal toxicity needs to be developed. We previously reported that an assessment using HCE-T cells reflected the corneal toxicity of ophthalmic solutions, and our findings were consistent those obtained in an in vivo study using rats as well as clinical data. ${ }^{5)}$ Therefore, we measured changes in the viability of HCE-T cells treated with 6 preservatives, and the stimulation time $(0-120 \mathrm{~s})$ was selected based on our previous study (Fig. 2). The viability of HCE-T cells was decreased by the treatment with the 6 preservations in the order of $\mathrm{BAC}>\mathrm{SC}>\mathrm{MP}>\mathrm{PP}>\mathrm{ZC}>\mathrm{BA}$. On the other hand, corneal toxicity was attenuated by the combination with mannitol, and the viabilities of HCE-T cells treated with mannitol for $120 \mathrm{~s}$ in combination with BAC, SC, MP, PP, ZC, and BA were 1.9-, 2.3-, 1.8-, 1.8-, 1.5-, and 1.7-fold higher, respectively, than those treated with the 6 preservatives alone. Liu et al. ${ }^{3)}$ reported that mannitol increased catalase levels and exerted antioxidant effects. Our previous studies using the hemolysis of rabbit red blood cells also showed that mannitol enhanced the endurance of the cell membrane, resulting in the inhibition of membrane disruption from the stimulation. ${ }^{4)}$ Therefore, the combination of BAC and mannitol will be important for the development of eye drop formulations with strong antimicrobial activity and low corneal toxicity. On the other hand, it is important to evaluate the association with long-term exposure as a further study.

In conclusion, we herein demonstrated that the addition of mannitol affected the antimicrobial activities and corneal toxicities of preservatives, and the balance between the contents of mannitol and preservatives in eye drop formulations is important. These results will serve as a guide for the future development of eye drop formulations.

Conflict of Interest The authors declare no conflict of interest.

\section{References}

1) Wilson L. A., Br. J. Ophthalmol., 80, 583-584 (1996). 2) Kim M. S., Choi C. Y., Kim J. M., Chang H. R., Woo H. Y., Br. J. Ophthalmol., 92, 1518-1521 (2008). 
3) Liu J. H., Chen M. M., Huang J. W., Wann H., Ho L. K., Pan W. H., Chen Y. C., Liu C. M., Yeh M. Y., Tsai S. K., Young M. S., Ho L. T., Kuo C. D., Chuang H. Y., Chao F. P., Chao H. M., J. Ocul. Pharmacol. Ther., 26, 249-257 (2010).

4) Nagai N., Yoshioka C., Tanino T., Ito Y., Okamoto N., Shimomura Y., J. Oleo Sci., 64, 743-750 (2015).

5) Nagai N., Ito Y., Okamoto N., Shimomura Y., J. Oleo Sci., 62, 159-166 (2013).

6) "The Japanese Pharmacopoeia," 17th ed. (JP17), Ministry of Health, Labour and Welfare, Tokyo, 2016.

7) See A. S., Salleh A. B., Bakar F. A., Yusof N. A., Abdulamir A. S., Heng L. Y., Am. J. Appl. Sci., 7, 620-627 (2010).

8) Bredin J., Davin-Régli A., Pagès J. M., J. Antimicrob. Chemother., 55, 1013-1015 (2005).
9) Nakagawa Y., Moldéus P., Biochem. Pharmacol., 55, 1907-1914 (1998).

10) Kim N. H., Park T. H., Rhee M. S., J. Appl. Microbiol., 116, $1447-$ 1457 (2014).

11) Sawai J., Kawada E., Kanou F., Igarashi H., Hashimoto A., Kokugan T., Shimizu M., J. Chem. Eng. Japan, 29, 627-633 (1996).

12) Debbasch C., Pisella P. J., De Saint Jean M., Rat P., Warnet J. M., Baudouin C., Invest. Ophthalmol. Vis. Sci., 42, 2525-2533 (2001).

13) De Saint Jean M., Brignole F., Bringuier A. F., Bauchet A., Feldmann G., Baudouin C., Invest. Ophthalmol. Vis. Sci., 40, 619-630 (1999).

14) Debbasch C., Brignole F., Pisella P. J., Warnet J. M., Rat P., Baudouin C., Invest. Ophthalmol. Vis. Sci., 42, 642-652 (2001). 